\title{
On the binding energy of atoms in crystals and the speed of sound
}

\author{
Aldo Dall'Osso* \\ 9 Avenue de Parthenay, 92400 Courbevoie, France
}

\begin{abstract}
The speed of sound depends on the structure and on the material properties of the crystal, such as the density and the Young's modulus. On the other hand, from atomistic arguments it is possible to link the Young's modulus to other material properties. These observations drive to a relation between the binding energy of the atoms in a crystal (which is one of the parameters appearing in the Mie-Lennard-Jones potential), the speed of sound in the longitudinal direction and the mass of one atom in the lattice. Applications of this relation to noble gases and few metals are presented and the results compared to others taken from literature.
\end{abstract}

Keywords: Binding Energy; Cohesive Energy; Mie Potential; Lennard-Jones Potential; Sound velocity

\section{Introduction}

The speed of sound in solids depends on the structure and on the material properties of the crystal. When the medium is a bar, the speed of sound $c_{s}$, in the longitudinal direction, can be expressed in terms of the ratio between the Young's modulus $Y$ and the density $\rho[1]$ :

$$
c_{s}^{2}=Y / \rho .
$$

On the other hand, the Young's modulus, defined as the ratio between the strain and the stress, can be derived from atomistic arguments $[2,3]$ based on the Mie-Lennard-Jones potential [4].

Combining the two expressions we obtain a relation between the binding energy and the speed of sound in the crystal, in the longitudinal direction. Its derivation is presented in Sec. 2 .

\footnotetext{
*Corresponding author. Tel.: +33 143336425

Email address: aldo.dallosso@free.fr (Aldo Dall'Osso)
} 


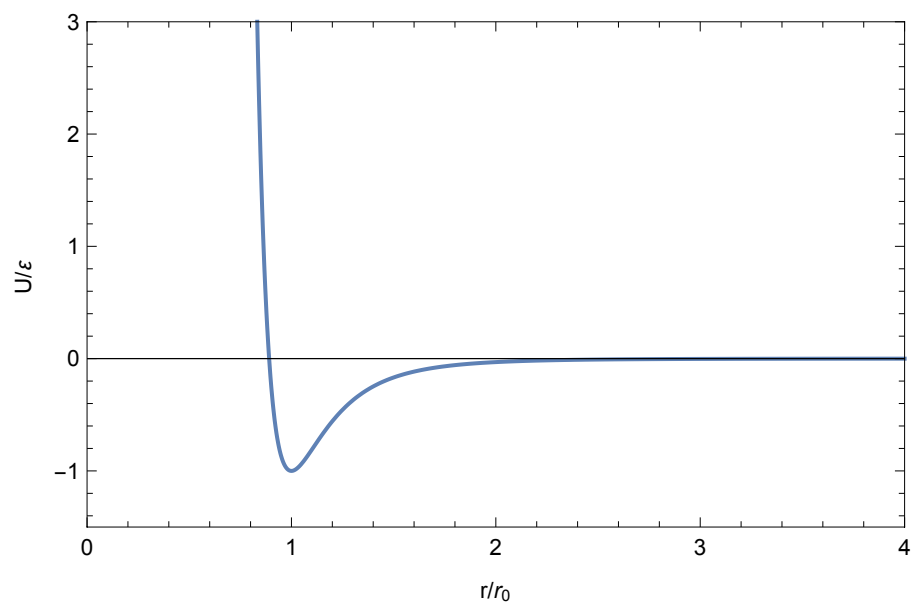

Figure 1: The Mie-Lennard-Jones potential.

\section{Theory}

\subsection{Young's modulus from Mie and Lennard-Jones potential}

The Mie potential is a simple particle-pair potential composed of two terms representing the repulsive and attractive forces between the particles. Following the approach proposed in refs. [2,3], it is used here to represent the forces acting between the atoms in the crystal. Its formulation is the following one $[5,6]$ :

$$
U(r)=\frac{\varepsilon}{m-n}\left(n\left(\frac{r_{0}}{r}\right)^{m}-m\left(\frac{r_{0}}{r}\right)^{n}\right),
$$

where $\varepsilon$ is the binding energy of the interacting particles (the energy required to separate them) and $r_{0}$ is the equilibrium distance between two particles. The exponents depend on the material.

A most used form, with $n=6$ and $m=12$, is the 12-6 Lennard-Jones potential [e.g. 7]:

$$
U(r)=\varepsilon\left(\left(\frac{r_{0}}{r}\right)^{12}-2\left(\frac{r_{0}}{r}\right)^{6}\right) .
$$

With reference to Fig. 1, which plots function $U(r)$, parameters $\varepsilon$ and $r_{0}$ are the depth of the potential $U(r)$ and its position, respectively.

The Young's modulus can be obtained from the definition of the Mie potential. Taking the derivative of the potential provides the force between the particles:

$$
F(r)=-\frac{m n}{(m-n)} \frac{\varepsilon}{r}\left(\left(\frac{r_{0}}{r}\right)^{m}-\left(\frac{r_{0}}{r}\right)^{n}\right),
$$




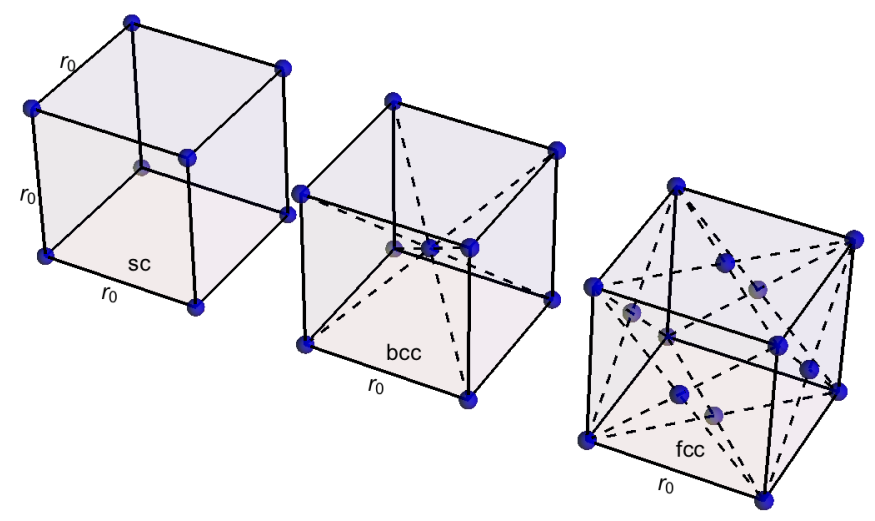

Figure 2: Cubic lattices: simple, body centered and face centered.

It can be seen that $F\left(r_{0}\right)=0$, which proves that for $r=r_{0}$ the potential $U(r)$ has a minimum. In the framework of our approach, $r_{0}$, which is the equilibrium distance, is also the size of the crystal cell as illustrated by the simple cube (sc) lattice in Fig. 2, representative of the internal structure of the bar.

Let us apply a force $F$ to the bar extremities. The atoms will be displaced from $r_{0}$ to $r$. Young's modulus $Y$ is defined as the ratio between stress (force per unit surface, $F(r) / r_{0}^{2}$ ) and strain (relative deformation, $\left.\left(r-r_{0}\right) / r_{0}\right)$ in the limit as the strain goes to 0. Therefore, in mathematical terms, Young's modulus can be written as:

$$
Y=\lim _{r \rightarrow r_{0}} \frac{F(r) / r_{0}^{2}}{\left(r-r_{0}\right) / r_{0}} .
$$

Taking into account that $F\left(r_{0}\right)=0$, Eq. (5) can be written as:

$$
Y=\frac{1}{r_{0}} \lim _{r \rightarrow r_{0}} \frac{\left(F(r)-F\left(r_{0}\right)\right)}{\left(r-r_{0}\right)},
$$

which, in virtue of the derivative definition, is equivalent to:

$$
Y=\left.\frac{1}{r_{0}} \frac{\mathrm{d} F(r)}{\mathrm{d} r}\right|_{r=r_{0}} .
$$

Computing the derivative of $F(r)$ from Eq. (4) we obtain:

$$
Y=m n \frac{\varepsilon}{r_{0}^{3}} .
$$




\subsection{Relation between binding energy and speed of sound}

From Eq. (1) and Eq. (8) we obtain:

$$
c_{s}^{2}=m n \frac{\varepsilon}{\rho r_{0}^{3}} .
$$

The term at denominator, $\rho r_{0}^{3}$, i.e. the density multiplied by the atom-cell volume, is the mass $M$ of a single atom-cell in a cubic lattice, where a simple cubic structure has been assumed, which enables writing:

$$
\rho=M / r_{0}^{3} .
$$

Substituting Eq. (10) into Eq. (9) provides the relation between binding energy and speed of sound in the longitudinal direction:

$$
\varepsilon=\frac{M c_{s}^{2}}{m n}
$$

Even if it has been assumed a simple cubic structure, this relation is valid for body centered (bcc) and face centered (fcc) cubic structures (see Fig. 2) as well (cf. Appendix A).

\section{Values of binding energy for several elements}

Eq. (11), has been used to compute the binding energy $\varepsilon$ for several elements having cubic structure, where the mass of the atom $M$ is defined as the ratio between the atomic weight $A$ and the Avogadro number $N_{\mathrm{Av}}$ :

$$
M=A / N_{\mathrm{Av}} .
$$

The elements studied are: 4 noble gases, $\mathrm{Ne}, \mathrm{Ar}, \mathrm{Kr}$ and $\mathrm{Xe}$, which at solid state have fcc crystal structure [8], 7 metals with fcc and 3 metals with bcc structure. Both the 12-6 Lennard-Jones and Mie potentials have been used. The data used in the formula, taken from references [9-13], is presented in Table 1.

\subsection{Values of binding energy using the 12-6 Lennard-Jones potential}

For this formulation Eq. (11) has been used with the values $n=6$ and $m=12$. The results for 4 noble gases are presented in Table 2 and compared to the ones obtained by Horton [6] solving a two-equation system set by fit of two crystal properties: the sublimation energy and the $0^{\circ} \mathrm{K}$ lattice size. The results for 8 metals are presented in Table 3 and compared to the ones obtained by Heinz et al. [7] and Kanhaiya et al. [14], who used a molecular dynamics 
Table 1: Data used in the evaluation of the binding energy for the elements studied.

\begin{tabular}{|c|c|c|c|c|}
\hline & $\begin{array}{l}\text { Atomic } \\
\text { Weight }\end{array}$ & $\begin{array}{l}\text { Crystal } \\
\text { Structure }\end{array}$ & $\begin{array}{l}\text { Speed of } \\
\text { Sound }(\mathrm{m} / \mathrm{s})\end{array}$ & Reference \\
\hline $\mathrm{Ne}$ & 20.149 & fcc & 1290 . & {$[9]$} \\
\hline $\mathrm{Ar}$ & 39.948 & fcc & 1630. & [10] \\
\hline $\mathrm{Kr}$ & 83.8 & fcc & 1335. & [11] \\
\hline $\mathrm{Xe}$ & 131.3 & fcc & 1150. & [12] \\
\hline A1 & 26.9815 & fcc & 6420. & [13] \\
\hline $\mathrm{Au}$ & 196.967 & fcc & 3240 . & [13] \\
\hline $\mathrm{Pb}$ & 207.2 & fcc & 2160. & [13] \\
\hline $\mathrm{Ni}$ & 58.6934 & fcc & 6040 . & [13] \\
\hline $\mathrm{Pt}$ & 195.078 & fcc & 3260 . & [13] \\
\hline $\mathrm{Ag}$ & 107.868 & fcc & 3650 . & [13] \\
\hline $\mathrm{Cu}$ & 63.546 & fcc & 4760. & [13] \\
\hline $\mathrm{Fe}$ & 55.845 & bcc & 5950 . & [13] \\
\hline Mo & 95.94 & bcc & 6250 . & [13] \\
\hline W & 183.84 & bcc & 5220 . & [13] \\
\hline
\end{tabular}

approach. The results for molybdenum and tungsten are also provided but no comparison is available.

Table 2: Binding energy (eV) for noble gases using the 12-6 Lennard-Jones potential.

\begin{tabular}{lll}
\hline & This work & Horton \\
\hline $\mathrm{Ne}$ & 0.00483 & 0.0045 \\
$\mathrm{Ar}$ & 0.01528 & 0.01473 \\
$\mathrm{Kr}$ & 0.0215 & 0.02028 \\
$\mathrm{Xe}$ & 0.025 & 0.02859 \\
\hline
\end{tabular}

\subsection{Values of binding energy using the Mie potential}

For this formulation Eq. (11) has been used with the values of $n$ and $m$ taken from Magomedov's work [5]. The results for 4 noble gases and 10 metals are presented in Tables 4 and 5, respectively, and compared to the ones obtained by Magomedov [5], who used an approach based on the preservation of measured quantities such as sublimation energy and thermal expansion coefficient. The approximation of interaction of only nearest-neighbor atoms has been adopted.

\section{Conclusion}

A relation between the binding energy in crystals and the speed of sound has been derived on the basis of the Mie and Lennard-Jones potentials. The 12-6 Lennard-Jones potential shows a quite good agreement with other results from literature, whereas the Mie potential shows higher differences, which can 
Table 3: Binding energy (eV) for 10 metals using the 12-6 Lennard-Jones potential.

\begin{tabular}{llll}
\hline & This work & Heinz et al. & Kanhaiya et al. \\
\hline $\mathrm{A} 1$ & 0.1601 & 0.1743 & - \\
$\mathrm{Au}$ & 0.2977 & 0.2294 & - \\
$\mathrm{Pb}$ & 0.1392 & 0.127 & - \\
$\mathrm{Ni}$ & 0.3083 & 0.245 & - \\
$\mathrm{Pt}$ & 0.2985 & 0.3382 & - \\
$\mathrm{Ag}$ & 0.2069 & 0.1977 & - \\
$\mathrm{Cu}$ & 0.2073 & 0.2046 & - \\
$\mathrm{Fe}$ & 0.2846 & - & 0.2601 \\
$\mathrm{Mo}$ & 0.5395 & - & - \\
$\mathrm{W}$ & 0.7211 & - & - \\
\hline
\end{tabular}

Table 4: Binding energy (eV) for noble gases using the Mie potential.

\begin{tabular}{lllll}
\hline & This work & Magomedov & $\mathrm{n}$ & $\mathrm{m}$ \\
\hline $\mathrm{Ne}$ & 0.0028 & 0.0045 & 5.83 & 21.39 \\
$\mathrm{Ar}$ & 0.01 & 0.015 & 6.62 & 16.69 \\
$\mathrm{Kr}$ & 0.0148 & 0.0205 & 6.56 & 15.92 \\
$\mathrm{Xe}$ & 0.0173 & 0.0285 & 6.73 & 15.42 \\
\hline
\end{tabular}

Table 5: Binding energy (eV) for 10 metals using the Mie potential.

\begin{tabular}{lllll}
\hline & This work & Magomedov & $\mathrm{n}$ & $\mathrm{m}$ \\
\hline $\mathrm{A} 1$ & 0.4239 & 0.5714 & 2.49 & 10.92 \\
$\mathrm{Au}$ & 0.7027 & 0.6387 & 1.96 & 15.56 \\
$\mathrm{~Pb}$ & 0.31 & 0.3399 & 2.27 & 14.24 \\
$\mathrm{Ni}$ & 0.8149 & 0.7506 & 3.56 & 7.65 \\
$\mathrm{Pt}$ & 0.6372 & 0.9795 & 2.53 & 13.33 \\
$\mathrm{Ag}$ & 0.4673 & 0.4944 & 3.08 & 10.35 \\
$\mathrm{Cu}$ & 0.5884 & 0.5895 & 3.03 & 8.37 \\
$\mathrm{Fe}$ & 0.8975 & 1.0838 & 3.54 & 6.45 \\
$\mathrm{Mo}$ & 2.3635 & 1.7042 & 2.14 & 7.68 \\
$\mathrm{~W}$ & 1.7695 & 2.2068 & 3.42 & 8.58 \\
\hline
\end{tabular}


reach $40 \%$. It is emphasized that this relation, expressed by Eq. (11), has only a theoretical purpose. It adopts some approximations such as to limit atoms interactions to nearest neighbors, therefore it is not supposed to be used for detailed calculations of interatomic interactions in crystals, where appropriate and accurate force fields have been derived. Moreover, these force fields are applicable to all kinds of crystals and to other forms of solid matter, whereas our approach is limited to crystals of pure elements.

The values of the binding energy computed for various elements depend on the form of the potential. Values associated to the 12-6 Lennard-Jones potential cannot be compared with the ones associated to the Mie potential. This is due to the fact that the values of the binding energy $\varepsilon$, the exponents $n$ and $m$, the $r_{0}$ distance, have to be seen as part of an inseparable whole.

\section{Acknowledgements}

I thank Prof. Hendrik Heinz (University of Colorado-Boulder, Boulder, USA) and Prof. Mahach N. Magomedov (Russian Academy of Sciences, Makhachkala, Russia) for their help in the interpretation of the results. I thank Prof. Joe Wolfe (School of Physics, UNSW Sydney, Bidjigal Country, Australia), author of reference [2], which is the source of inspiration of this work.

\section{Appendix A. Case of face and body centered cubic structures}

Eq. (11), expressing the relation between the binding energy and the square of speed of sound, has been obtained in case of simple cubic structure. We show here that the cases of face centered (fcc) and body centered (bcc) cubic structures respond to the same relation.

In case of fcc or bcc structures (Fig. 2), the lattice can be seen as $N_{\text {Lat }}$ embedded lattices with cells of size $r_{0}$, where one of the lattices is related to the vertices and:

- three others to the center of the faces, in case of fcc,

- the other one to the center of the body, in case of bcc.

Summarizing, $N_{\text {Lat }}$ is 4 for fcc and 2 for bcc. Therefore the surface $r_{0}^{2}$ is affected by $N_{\text {Lat }}$ pairs of interacting particles and the force appearing in Eq. (4) has to be multiplied by $N_{\text {Lat }}$, which conducts to a Young modulus $N_{\text {Lat }}$ times higher than in the case of simple cubic structure:

$$
Y=N_{\text {Lat }} m n \frac{\varepsilon}{r_{0}^{3}} .
$$

This means that the square of the speed of sound, which is proportional to $Y$ according to Eq. (1), is $N_{\text {Lat }}$ times higher too and Eq. (9) becomes:

$$
c_{s}^{2}=N_{\text {Lat }} \frac{m n \varepsilon}{\rho r_{0}^{3}} .
$$


On the other hand, in a fcc or bcc lattice cell there is more than one atom. This number is $N_{\text {Lat }}$, which drives to replace Eq. (10) by the following one:

$$
\rho=N_{\text {Lat }} M / r_{0}^{3} .
$$

Substituting Eq. (A.3) into Eq. (A.2) and solving with respect to $\varepsilon$ we obtain the relation between binding energy and speed of sound:

$$
\varepsilon=\frac{M c_{s}^{2}}{m n},
$$

which is the same as Eq. (11).

\section{References}

[1] L.E. Kinsler, A.R. Frey, A.B. Coppens, J.V. Sanders, Fundamentals of Acoustics, 4th edition, John Wiley \& Sons, 1999.

[2] J. Wolfe, Young's modulus, Hooke's law and material properties. UNSW Sydney, Australia; https://www.animations.physics.unsw.edu.au/jw/elasticity.htm

[3] D. Roylance, Mechanics of Materials, John Wiley \& Sons, 1996.

[4] A. Stone, The theory of intermolecular forces. Oxford University Press (UK), 2013.

[5] M.N. Magomedov, The Calculation of the Parameters of the Mie-LennardJones Potential, High Temperature 44(4) (2006) 513-529.

[6] G.K. Horton, Ideal Rare-Gas Crystals, American Journal of Physics 36(2) (1968) 93.

[7] H. Heinz, R.A. Vaia, B.L. Farmer, R.R. Naik, Accurate Simulation of Surfaces and Interfaces of Face-Centered Cubic Metals Using 12-6 and 9-6 LennardJones Potentials, J. Phys. Chem. C 112(44) (2008) 17281-17290.

[8] K.F. Niebel, J.A. Venables, An explanation of the crystal structure of the rare gas solids. Proc. R. Soc. Lond. A. 336 (1974) 365-377.

[9] T.M. Hakim and H.R. Glyde, Dynamics of solid neon monolayers. Physical Review B, 37(2) (1988) 984.

[10] D.J. Lawrence, F.E. Neale, Longitudinal wave velocity in solid argon. Proceedings of the Physical Society (1958-1967) 85(6) (1965) 1261.

[11] H. Peter, P. Korpiun, E. Lüscher, Measurement of the longitudinal sound velocity in solid krypton at $4.2^{\circ} \mathrm{K}, 77^{\circ} \mathrm{K}$ and $90^{\circ} \mathrm{K}$, Physics Letters A $26(5)$ (1968) 207.

[12] P.L.R. Weigel, E.V. Hansen, M.J. Dolinski, Development and Characterization of Solid Noble Bolometers (2019) Arxiv-1910.06276.

[13] D.R. Lide ed., CRC Handbook of Chemistry and Physics, CRC Press, Boca Raton, FL, 2005.

[14] K. Kanhaiya, S. Kim, W. Im, H. Heinz, Accurate simulation of surfaces and interfaces of ten FCC metals and steel using Lennard-Jones potentials, npj Computational Materials 7(1) (2021) 1-15. 